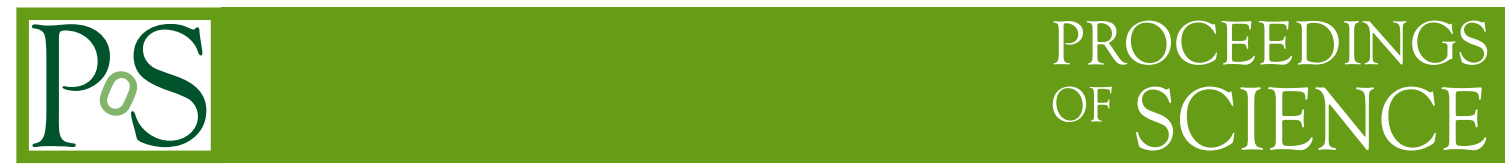

\title{
Neutrino astrophysics at ICRC 2017
}

\author{
Markus Ackermann ${ }^{* \dagger}$ \\ $\dagger$ DESY, D-15738 Zeuthen, Germany \\ E-mail: markus.ackermann@desy.de
}

High-energy neutrino astrophysics is one of the youngest fields of astrophysics. Until very recently, only the sun and SN 1987a were established astrophysical sources of neutrinos at MeV energies. With the discovery of cosmic $\mathrm{TeV}$ and PeV neutrinos in 2013, a new high-energy window into the universe has opened that promises exciting new insights into the non-thermal universe for the years and decades to come. A selection of the contributions to the ICRC 2017 in the field of neutrino astrophysics is presented here, based on the corresponding rapporteur talk at the conference venue in Busan.

35th International Cosmic Ray Conference - ICRC2017

10-20 July, 2017

Bexco, Busan, Korea

${ }^{*}$ Speaker. 


\section{Introduction}

\subsection{Year 4 of high-energy neutrino astrophysics}

The discovery of high-energy cosmic neutrinos in 2013 using the IceCube neutrino telescope [1] started a new era of multi-messenger astrophysics and allowed a new and unique view into the non-thermal universe. Until then, only the sun [2, 3] and SN1987 [4] were known cosmic sources of neutrinos in the $\mathrm{keV}-\mathrm{MeV}$ energy range. The neutrinos in both sources are produced in nuclear processes. In contrast, the neutrinos observed by IceCube at $\mathrm{TeV}$ to $\mathrm{PeV}$ energies originate from the interactions of non-thermal populations of protons and nuclei, the cosmic rays (CRs). Undeflected by magnetic fields and unabsorbed by even dense matter or intense radiation fields, they can reach us from their production sites throughout the universe, and give us insights about the mechanisms and environments that accelerate CR to such astonishing energies.

In the first years after the discovery, great progress has been made in studying the spectrum and flavor composition of the cosmic TeV-PeV neutrinos [5]. Yet, no individual source or correlation to a known source population had been clearly identified. Many new studies focused on the origin of the astrophysical neutrinos have been presented at this conference, still not finding striking evidence for their origin. It becomes more and more evident that a new generation of instruments is needed to solve the puzzle, extending the sensitivity of current instruments to a larger fraction of the sky, dimmer sources, and a wider energy range. This future generation of instruments is taking shape at a fast pace, as evidenced by the many design studies, R\&D and construction efforts presented at the ICRC 2017.

\subsection{The uniqueness of neutrinos as an astrophysical messenger}

Neutrinos are indeed a unique astrophysical messenger in many respects. Their low interaction cross-section allows them to escape environments of dense matter or radiation fields. They can reach us from deep within stars, can penetrate the expanding stellar hull during supernova explosions, and escape the intense photon fields of the accretion disks of active galactic nuclei (AGN).

As one goes to higher and higher energies the universe loses its transparency to electromagnetic radiation. Electron pair-production of high-energy gamma rays with the extragalactic photon fields that are present everywhere in the universe creates a horizon beyond which photons are strongly absorbed. This horizon gets closer as the energy increases. While we can observe the universe well beyond the peak of star formation with photons at $\mathrm{GeV}$ energies, only the local universe is visible at $\mathrm{TeV}$ energies. At $\mathrm{PeV}$ the horizon is at the distance of our own Galactic center, rendering extragalactic astronomy with electromagnetic radiation at these energies impossible. Only neutrinos allow us to peek into the distant universe above few tens of $\mathrm{TeV}$.

Neutrinos are also a diagnostic of the interactions of hadrons in cosmic sources, as the astrophysical production processes of high-energy neutrinos inadvertently involve nucleons, scattering off nucleon or photon targets. Electromagnetic radiation is produced in a variety of processes involving the interactions of both electrons and hadrons. Therefore, a neutrino source could be directly identified as a site of cosmic-ray acceleration and resolve the long-standing puzzle about their origin. While gamma-ray observations have delivered striking spectral signatures for cosmicray acceleration in middle-aged supernova remnants [6] up to $\mathrm{TeV}$ energies, it is still unknown 
where the Galactic PeV CRs as well as the ultra-high-energy cosmic rays (UHECR) beyond PeV energies are produced that are commonly considered to be of extragalactic origin.

Finally, MeV neutrinos from a Supernova ( $\mathrm{SN}$ ) explosion inside our Galaxy could revolutionize our understanding of core-collapse SNe. 30 years after the first detection of neutrinos from SN1987a, we have several neutrino detectors and telescopes world-wide in place to observe a new explosion with unprecedented statistics. With the next generation of underground neutrino detectors also the detection of the SN neutrino background - the quasi-diffuse flux produced by all $\mathrm{SNe}$ in the universe - will be in the reach of observations.

\subsection{Neutrino astrophysics at the ICRC2017}

This great potential of neutrinos as an astrophysical messenger was reflected in the many excellent contributions from the field of neutrino astrophysics to this ICRC. 3 highlight talks, 55 parallel talks and 79 posters have been presented. Many new results have been shown concerning the measurement of the properties of the cosmic neutrino flux, as well as searching for its origin. About one third of the contributions discuss the properties and potential origins of the astrophysical neutrinos. Almost 50 contributions were directed towards future instrumentation, making a strong statement that neutrino astrophysics is a growing field.

In the following sections, I will attempt to highlight some of these contributions. My selection of highlights is naturally very subjective. All neutrino astrophysics studies presented at the ICRC were of very high quality and worth mentioning here, but unfortunately it is impossible to give credit to 137 contributions in such a short summary. The next section addresses the progress of our understanding of origin and properties of the high-energy neutrino flux discovered with the IceCube neutrino telescope. In Section 3 the searches for $\mathrm{MeV}$ neutrinos from $\mathrm{SNe}$ and the sun are discussed. Section 4 summarizes the efforts for new instrumentation, before a short conclusion is presented in Section 5.

\section{Origin and properties of the cosmic TeV-PeV neutrino flux}

\subsection{Measurements of the energy spectrum \& flavor composition}

The IceCube neutrino observatory [7] located at the geographic South Pole is currently the only instrument that can detect the cosmic neutrino signal with high significance. Hence, the measurement of its properties are based on the data that IceCube is collecting since its completion in 2011, and during the construction phase before. In comparison to the results shown at the ICRC 2015, the amount of data analyzed approximately doubled, allowing more precise measurements of spectrum and flavor composition.

With its 5160 optical sensors deployed in $\sim 1 \mathrm{~km}^{3}$ of Antarctic ice, IceCube detects neutrinos through the Cherenkov light that is produced by charged secondaries after a neutrino interaction. Accordingly, the neutrino interactions are classified into different topologies based on the light detection patterns. Track-like events arise from charged-current (CC) $v_{\mu}$ interactions outside of the detector, where the muon produced in the interaction travels through the instrumented volume of IceCube, leaving a long trail of Cherenkov photons. Shower-like events, with a rather spherical distribution of Cherenkov photons around the interaction vertex, arise from neutral current (NC) interactions of all flavors, as well as from CC interactions of $v_{e}$ and low-energy $v_{\tau}$ 
$(\mathrm{E} \lesssim 100 \mathrm{TeV})$. Starting tracks are charged-current (CC) $v_{\mu}$ interactions inside the detector, where both, the hadronic shower at the interaction vertex and the track produced by the muon, are visible. Shower-like events well inside the instrumented volume and starting tracks together are often denoted as starting events. And finally, some high-energy $v_{\tau}(\mathrm{E} \gtrsim 100 \mathrm{TeV})$ can produce doublebang events. Here the hadronic shower at the $v_{\tau}$ interaction vertex and the shower at the $\tau$ decay vertex are separated far enough to individually resolve these two showers in IceCube.

At the ICRC 2015, the IceCube collaboration had presented a combined analysis of showerlike events, starting events and track-like events to determine spectrum and flavor composition of the cosmic neutrino flux [8]. The spectrum was found to be compatible with a single power law with an index of $\gamma=2.5$ between $20 \mathrm{TeV}$ and $3 \mathrm{PeV}$. Alternatively, it could be described by a harder power-law with index $\gamma=2.3$ and an exponential cutoff at $3 \mathrm{PeV}$. The flavor composition found was compatible with the $v_{e}: v_{\mu}: v_{\tau}=1: 1: 1$ flavor ratio expected from pion production in astrophysical sources.
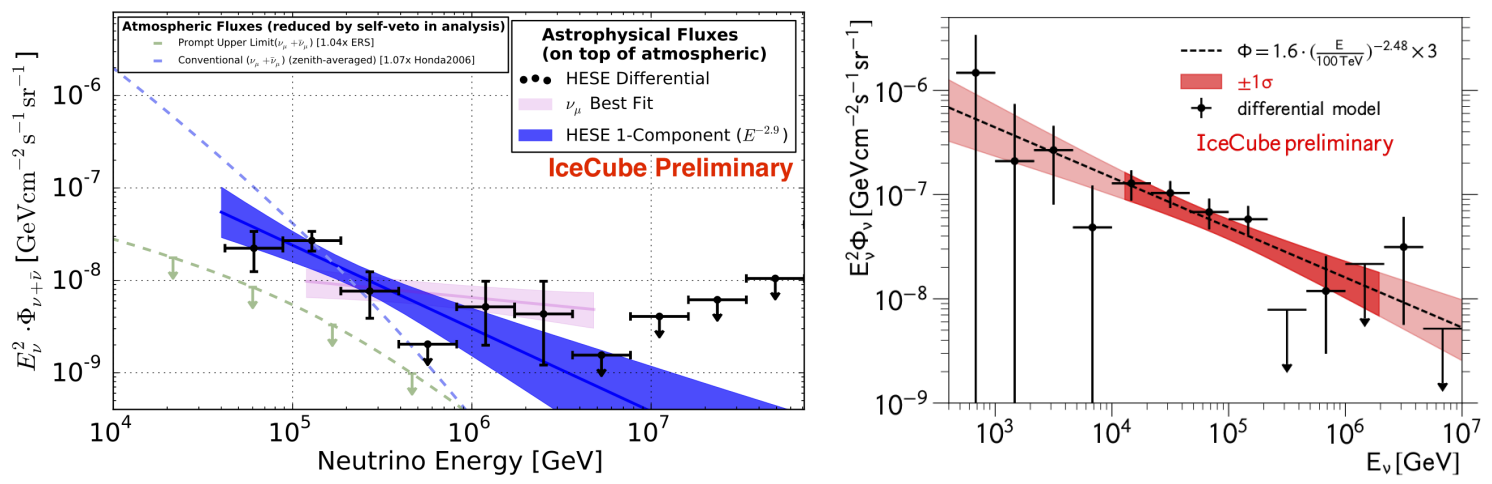

Figure 1: Left: Spectrum of cosmic neutrinos from 6 years of high-energy starting events, fitted in individual energy bands (black crosses) and with a power-law hypothesis (blue band). The best-fit power law from track-like events collected in 8 years is given by the red band. Figure taken from [9]. Right: Spectrum of cosmic neutrinos derived from a 4-year sample of shower-like events, fitted in individual energy bands (black crosses) and with a power-law hypothesis (red band). Figure taken from [10].

At this conference we have seen updates of the individual detection channels that provided the input for the combined analysis, with a substantial increase in the amount of data analyzed. An 8year dataset of track-like events has been analyzed [11], as well as a 6-year dataset of high-energy starting events [9], and an additional 4 years of shower-like events [10]. The three panels in Figure 1 summarize the spectra derived from these individual channels. Interestingly, a rather soft spectrum is seen for the shower-type events $(\gamma=2.5 \pm 0.1)$ and the high-energy starting events $(\gamma=2.9 \pm$ $0.3)$, while the spectrum for the track-like events is substantially harder $(\gamma=2.2 \pm 0.1)$. This is not necessarily a tension in the measurements, as the energy range where each of these measurements is sensitive to the astrophysical neutrino flux varies by more than an order of magnitude. The lower bound is at $\sim 10 \mathrm{TeV}$ for shower-like events, but at $\sim 120 \mathrm{TeV}$ for track-like events. So it might be an indication that the astrophysical neutrino flux shows a spectral hardening at energies around $100 \mathrm{TeV}$. An updated combined analysis is necessary to derive a quantitative statement how significant such a spectral feature would be, as these datasets cannot be trivially combined (they are not independent and have correlated systematic uncertainties). The expected performance of such 
a combined analysis has been shown in [12], but no results have been presented yet.

Flavor composition studies have been improved by searching for the typical double-bang signature of a high-energy $v_{\tau}$ in the starting event sample. As mentioned above, for a separation of more than $20 \mathrm{~m}$ between the generation and decay vertices of the $\tau$, two individual showers can be identified. A new study on the 6-year dataset of high-energy starting events has found a way to isolate these signatures, and expected to identify $1.4 v_{\tau}$ interactions in this sample on a background of 0.94 events (assuming a $v_{e}: v_{\mu}: v_{\tau}=1: 1: 1$ flavor composition). The background arises from $v_{\mu}$ and $v_{e}$ interactions that can mimic a double-bang signature in combination with the complex light propagation in natural ice. No events have been found that can be classified as 'double-bang' events in the investigated sample [13]. This is compatible with a statistical fluctuation for the total expected number of 2.4 events. Figure 2 shows the flavor composition constraints that arise from this analysis in comparison to the combined analysis from 2015. The standard astrophysical scenarios are compatible with this non-observations. Future analysis will focus on expanding the search to shower-type events [14], in particular also events that happen outside of the instrumented volume [15], increasing the rate of identifiable $v_{\tau}$ by up to $50 \%$, and will hopefully lead to the first detections of $v_{\tau}$ candidates.

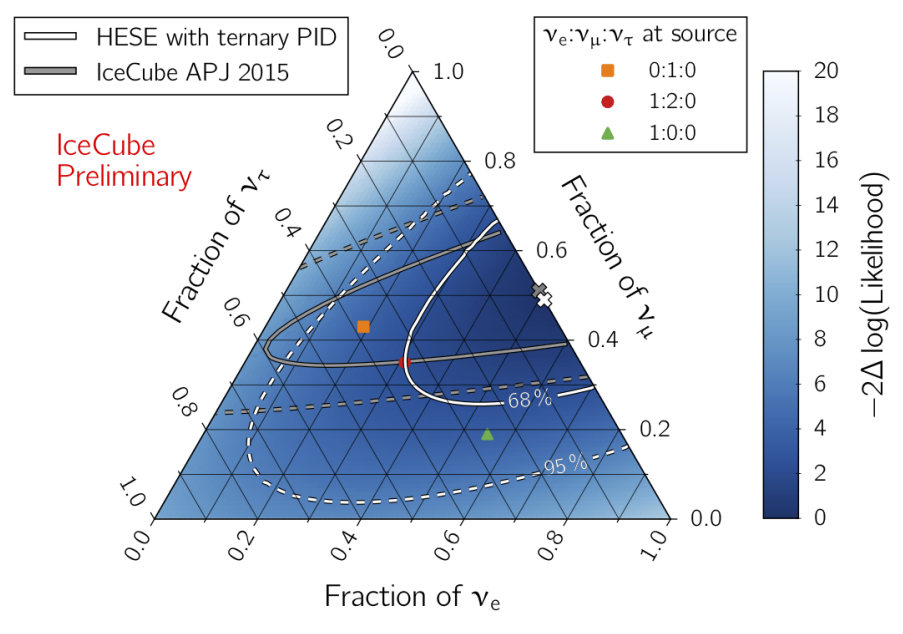

Figure 2: Flavor composition constraints from a search for $v_{\tau}$ signatures in a 6-year sample of IceCube high-energy starting events (white contours). The flavor composition constraints from a previous combined analysis of multiple IceCube event signatures without $v_{\tau}$ identification [5] are indicated as gray contours for comparison. Figure taken from [13].

An interesting new concept for improved flavor identification has been presented in [16]. As suggested in [17], a Cherenkov light "echo" from the capture of neutrons produced in a hadronic shower, might be used to distinguish them from electromagnetic showers. A new, dedicated readout concept had to be developed and installed for the IceCube detector to search for these light echos, as they are expected with an about $100 \mu$ s delay. This dedicated readout has been working since February 2016. Figure 3 shows the cumulative signal from a sample of events that is dominated by atmospheric muon background. A clear neutron capture echo is visible, likely originating from photonuclear interactions of the high-energy muons in the sample. 


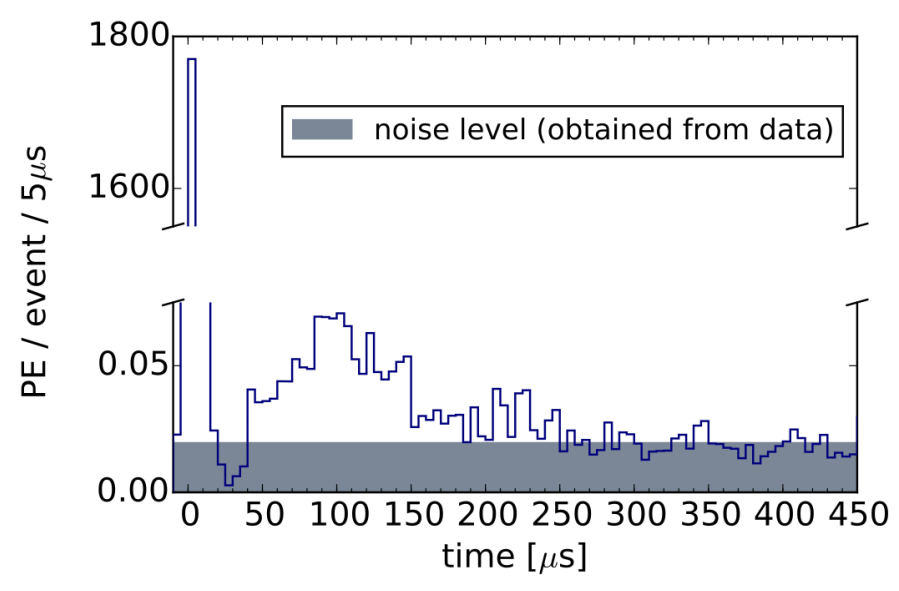

Figure 3: Stacked time distribution of Cherenkov photons detected with IceCube from 1250 high-energy starting events, each depositing between 1500 and 6000 photo-electrons in IceCube's optical modules. Figure taken from [16].

\subsection{Searches for the origin of the cosmic neutrinos}

The most pressing question in neutrino astrophysics about the origin of the cosmic neutrinos remains unsolved. 34 contributions at this ICRC alone were attempting to resolve the sources that produce the observed high-energy neutrinos. This quest is not an exclusive IceCube domain. ANTARES is an underwater neutrino telescope off the coast of Southern France [18]. Working on the same detection principles as IceCube, the instrumented volume is more than an order of magnitude smaller. Nevertheless, due to its location in the Northern Hemisphere, it is more sensitive than IceCube for a large fraction of the Southern sky, in particular for neutrinos below $\mathrm{E} \lesssim 100 \mathrm{TeV}$. At very high energies ( $\mathrm{E}>100 \mathrm{PeV}$ ), the Pierre Auger Observatory (PAO) [19] contributes, too. Built to measure the air-showers of UHECR, it can also detect air showers from the decays of $\tau$ that are produced by $v_{\tau}$ interactions in the Earth or nearby mountain ranges [20], and reach a comparable sensitivity to IceCube at the highest energies.

Both ANTARES and IceCube searched for point and spatially extended sources of neutrinos. In this search, the atmospheric neutrinos form a diffuse background over which cosmic neutrino sources would stand out as an excess of events, localized on a specific point in the sky. The shape of this excess corresponds to the point spread function (PSF) of the instrument for point sources. For an extended sources the shape would be a convolution of the source shape (usually assumed as a Gaussian intensity profile) with the instrument PSF. Neither ANTARES nor IceCube found such an excess that is statistically significant, neither for point sources [21] nor for extended sources [22]. Correspondingly, flux upper limits have been set, as shown in Figure 4.

The search described above would not be the most sensitive way to identify short transients of neutrinos. However, many candidate sources of high-energy cosmic rays and neutrinos are such short violent transients, e.g., gamma-ray bursts, SN explosions, tidal disruption events or the flares of AGN. Often these transients are observed by their electromagnetic emission in various frequency bands, or, recently by their gravitational wave emission [25]. Searching for neutrinos correlated in 


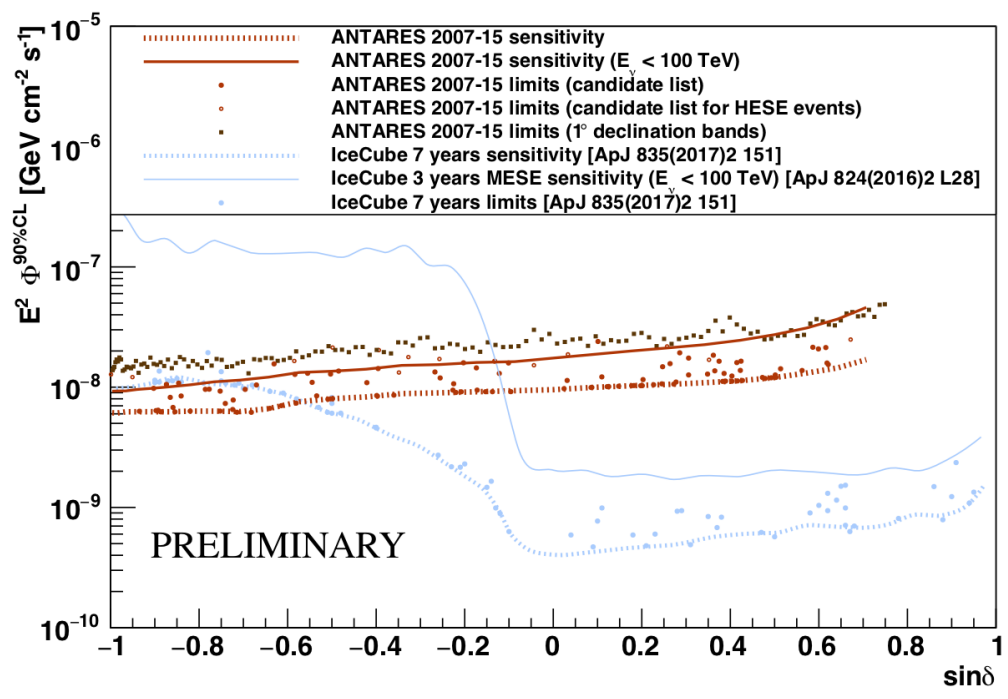

Figure 4: ANTARES upper limits at $90 \%$ C.L. on the all-flavor neutrino flux of investigated source candidates, assuming a power-law spectrum with index $\gamma=2$ (red circles). The dashed red line shows the ANTARES sensitivity at the corresponding declination band, the solid red line shows the corresponding sensitivity if the sources have a maximum neutrino energy of $\mathrm{E}=100 \mathrm{TeV}$. Dark red points correspond to the flux upper limit derived from the cluster with the largest excess of events in each $1^{\circ}$ declination band. Corresponding point source limits and sensitivities from IceCube are shown in blue [23, 24]. Figure taken from [21].

space and time with these transients can greatly reduce the background from atmospheric neutrinos and boost the sensitivity of source searches. A number of such searches has been presented at this ICRC. No significant correlation of neutrinos has been observed by either ANTARES or IceCube with gamma-ray bursts [26], blazar flares [27, 28, 29, 30], fast radio bursts [31, 32], gravitational wave events [33], x-ray flares [34], x-ray binaries [35] or SNe [36]. The ANTARES collaboration also checked for a correlation of the neutrino events observed in their detector, with the high-energy neutrinos observed in IceCube, also producing a null result [37].

A final possibility to find the origin of the cosmic neutrino fllux in the case that individual sources are too dim to show up individually as significant detections, are cross-correlation searches with known candidate source catalogs, and autocorrelation searches that would be sensitive to small-scale anisotropies from the combined emission of many weak neutrino sources. The IceCube collaboration has presented a cross-correlation search looking for a correlation between IceCube neutrinos and several gamma-ray and candidate gamma-ray blazar catalogs (the 3LAC [38], 2FHL [39], 2WHSP [40] catalogs). No correlation with any of these catalogs has been found, limiting the contribution of the sources in these catalogs to a few \% of the observed IceCube neutrino flux [41]. An analysis of the two-point autocorrelation function of the observed IceCube neutrinos did also not yield any small-scale anisotropy hidden in the data [42]. The ANTARES collaboration demonstrated in a study on x-ray selected blazars how such correlation studies improve the discovery potential [43].

In [44] a different type of correlation was investigated. Assuming that the neutrinos would 
be produced in the same sources as the UHECRs, one could expect a directional correlation to the cosmic rays observed by PAO and the Telescope Array (TA) [45], at least in the cases where the chemical composition of the cosmic rays and the intergalactic magnetic fields would yield relatively low deflection angles. A former study had found an indication of such a correlation between the shower-like IceCube high-energy events and UHECR on a $\sim 3 \sigma$ significance level [46]. The same study has been presented here with new data from IceCube, PAO and TA added to the respective datasets [44]. The correlation significance dropped to about $2 \sigma$, suggesting that the earlier result was a statistical fluctuation rather than a genuine correlation. This is also supported by the lack of correlation for track-like IceCube events, and the non-observation of a significant correlation of ANTARES events to cosmic rays [47].

The observed cosmic neutrinos could also be genuinely diffuse in nature. A guaranteed production mechanism of $\mathrm{TeV}$ neutrinos is the interaction of cosmic rays propagating through the Milky Way and interacting with interstellar gas. While there is a general correlation expected for the produced neutrinos with the Galactic plane, different propagation models yield different spectral and spatial distributions of the expected neutrinos. As ANTARES is ideally positioned to observe the central part of the Galaxy located in the Southern sky, both IceCube and ANTARES can search for an emission of neutrinos from the Galactic plane with similar sensitivity [48, 49, 50]. No apparent correlation to predictions of the spatial distribution of Galactic neutrinos is found. This implies that only a small fraction of the neutrino flux observed with IceCube can originate from cosmic-ray interactions in our Galaxy, however, the obtained upper limits on the Galactic flux shown on Figure 5 are still slightly above even optimistic predictions [51].

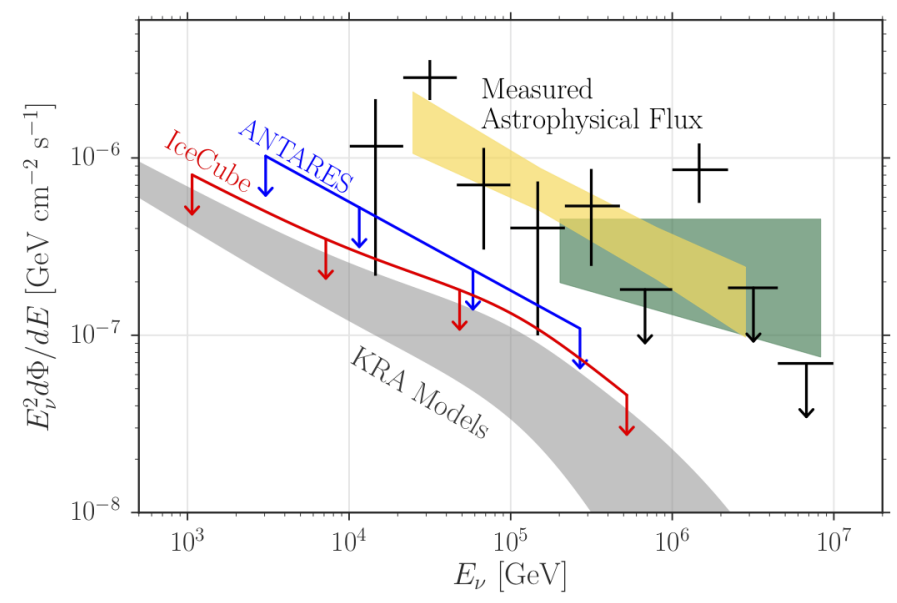

Figure 5: IceCube and ANTARES upper limits on the all-flavor (1:1:1 flavor ratio assumption) neutrino flux from the Galaxy with respect to KRA model predictions [51], and the measured astrophysical neutrino flux. Figure taken from [49].

At very high energies a diffuse flux of neutrinos could be created by photo-hadronic interaction of UHECR with the CMB and other radiation fields present throughout the universe. As UHECR are considered to be of extragalactic origin, this flux of neutrinos would be highly isotropic. However, due to the frequency dependent energy densities in the universe's radiation field, the energy 
flux of neutrinos is expected to be peaked at $\mathrm{EeV}$ energies, beyond the range where the cosmic neutrino flux is observed by IceCube. Neither IceCube nor PAO observe any neutrinos at these energies [20,52], providing similar upper limits on this so-called "cosmogenic" neutrino flux. Figure 6 shows the current IceCube and PAO flux upper limits presented at this ICRC. A candidate neutrino event with an energy of several hundred $\mathrm{PeV}$ was discovered by a different experiment. ANITA, a balloon-borne neutrino detector designed to detect the radio emission from particle showers produced in neutrino interactions [53], has observed the candidate event on one of their circumAntarctic flights [54]. However, interpreting this event as a genuine neutrino interaction causes some tension with the IceCube and PAO limits that have a superior sensitivity in this energy range and not observed any events.
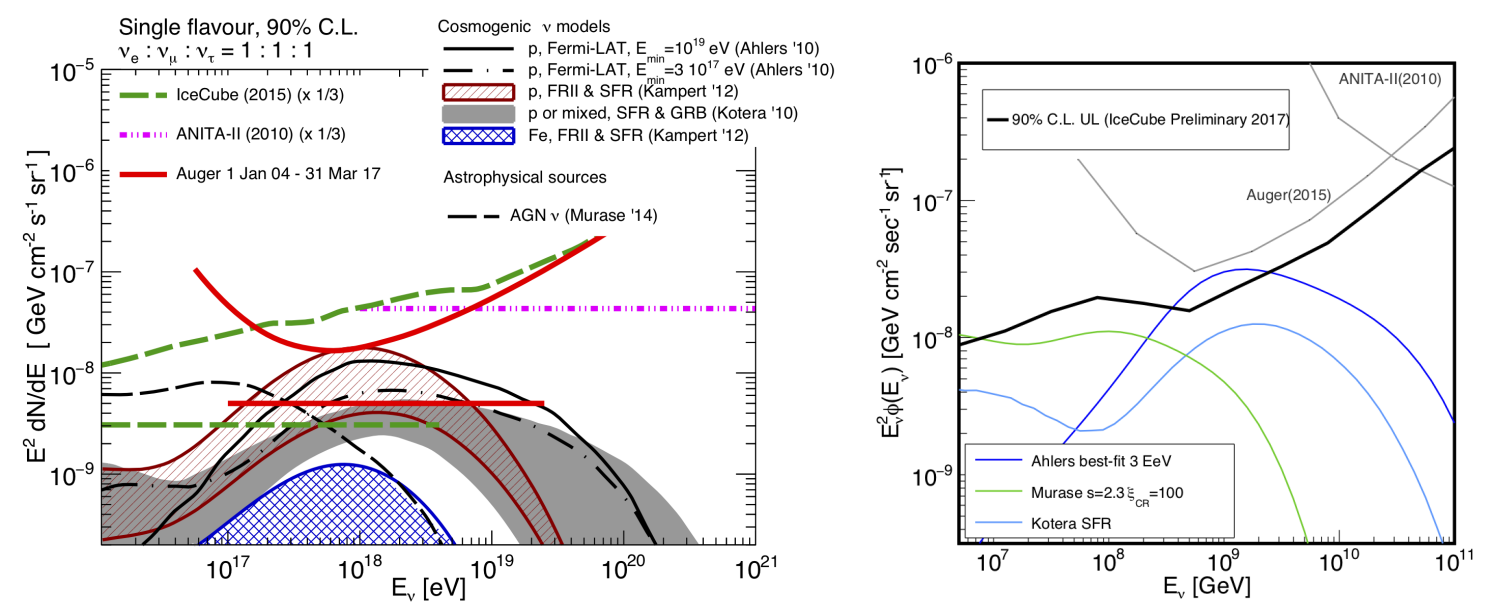

Figure 6: Left: Integral and differential flux upper limits (at 90\% C.L.) from PAO observations for an isotropic neutrino flux. Limits are quoted for a single flavor assuming equal flavor ratios. IceCube and ANITA upper limits are given for comparison, as well as several predictions of the astrophysical and cosmogenic neutrino flux. Figure taken from [20]. Right: Updated differential limit from a search for cosmogenic neutrinos with IceCube. Here the limit is quoted for the sum flux from all neutrino flavors. Figure taken from [52].

\subsection{Modeling cosmic neutrino sources and atmospheric backgrounds}

An essential part to connect the observations to physics processes in potential neutrino sources, is the modelling of the environments in which they can be produced. In addition, a good understanding of the atmospheric backgrounds is critical for the observers. A number of interesting works has been presented at this conference describing the phenomenology of potential sources, as well as of the expected atmospheric backgrounds.

Gamma-ray bursts have been modelled accelerating heavy nuclei in [55]. The full nuclear cascade is taken into account to predict spectrum and composition of cosmic rays and the expected neutrino fluences, and compare them to observations of UHECR and flux upper limits from IceCube. This can be a powerful tool to constrain modelling parameters, as part of the phase space allowed by $\mathrm{CR}$ observations can be excluded from the non-observation of coincident neutrinos with GRB (cfr. Figure 7). 


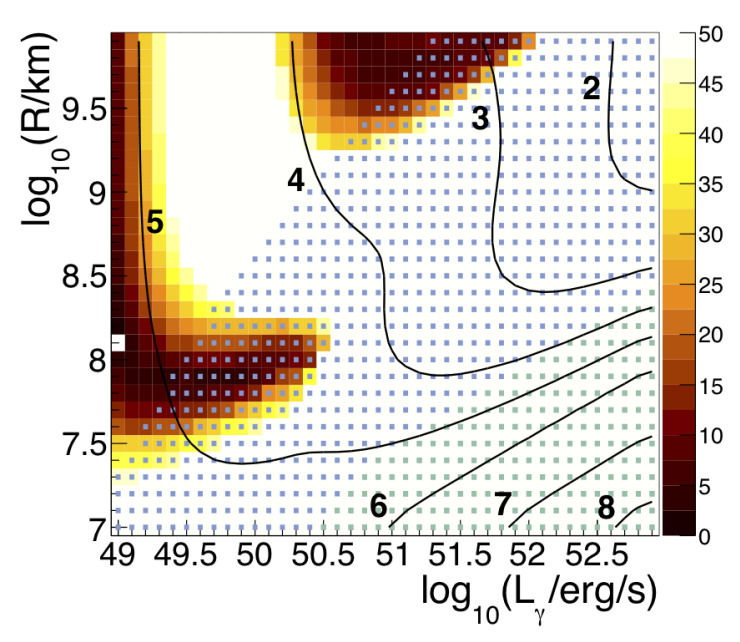

Figure 7: Parameter space scan for the Mixed Composition Ankle Model in the internal shock scenario. On the color axis, $\Delta \chi^{2}$ is shown for the fit to cosmic-ray data of the PAO above $10^{19} \mathrm{eV}$, including a penalty for the overshooting of the flux at lower energies. The parameters of the model are the collision radius $\mathrm{R}$ of the internal shocks, and the gamma-ray luminosity $\mathrm{L}_{\gamma}$ of the GRBs. Blue and green squares mark the current (90\% C.L.) IceCube-excluded region from GRB stacking and cosmogenic neutrino analysis, respectively. The contours show the logarithm of the nuclear loading factor (see [55] for more details). Figure taken from [55].

An interesting new model of neutrino production in AGN has been presented in [56]. Here, neutrons from jet accelerated nuclei escape from the jet and interact with gas in the accretion disk, producing neutrinos and gamma rays. As the gamma rays get absorbed in the intense accretion disk radiation fields, these sources would be gamma-ray 'dark', a desired feature to avoid tensions between the observed neutrino and extragalactic gamma-ray fluxes [57].

A precision calculation of the atmospheric neutrino flux has been presented in [58]. In particular, the CR flux model has been updated compared to an older version of this calculation, including new measurements, e.g., from AMS-02 [59,60]. A 5\% flux decrease is found around $10 \mathrm{GeV}$ in energy. This is an important energy range for studies of neutrino oscillation properties.

A new approach to fit the cosmic-ray composition was presented in [61, 62]. With minimal assumptions on the spectrum, the fluxes of several element groups were fitted against selected direct and indirect CR measurements. In this procedure not only a best-fit CR composition and spectrum was obtained, but also the respective uncertainties. The fitted parameters imply a several times higher prompt neutrino flux at $\mathrm{PeV}$ energies than what was previously estimated.

\section{MeV neutrinos from core-collapse supernovae and the sun}

\subsection{MeV neutrino astronomy}

In contrast to the fast-developing new field of high-energy astrophysics, $\mathrm{MeV}$ neutrino astronomy is characterized by patient observations of two sources, the sun and supernovae. The focus is on ever more precise observations of the sun, keeping the detectors ready for the next Galactic SN, 
and upgrading instrumentation to be able to observe the SN relic background from all the distant $\mathrm{SNe}$ in the universe.

The Super-Kamiokande (SuperK) detector in Japan [63] is one of the central instruments in this field. It consists of a large tank of ultra-pure water lined with Photomultipliers (PMTs) all over its inner surface to detect the Cherenkov light from neutrino interactions in the water. The rich data from the observation of a Galactic core-collapse SN with SuperK and other neutrino detectors around the world would certainly revolutionize our understanding of stellar death. In the mean time we see interesting observations of the sun in neutrinos. To date more than 50,000 neutrinos have been observed from the sun by SuperK, and among other studies their arrival time structure has been analyzed thouroughly. An interesting indication of a weak periodicity in the signal from the first phase of SuperK operations, was not confirmed with independent SuperK data from their latest phase of operations [64].

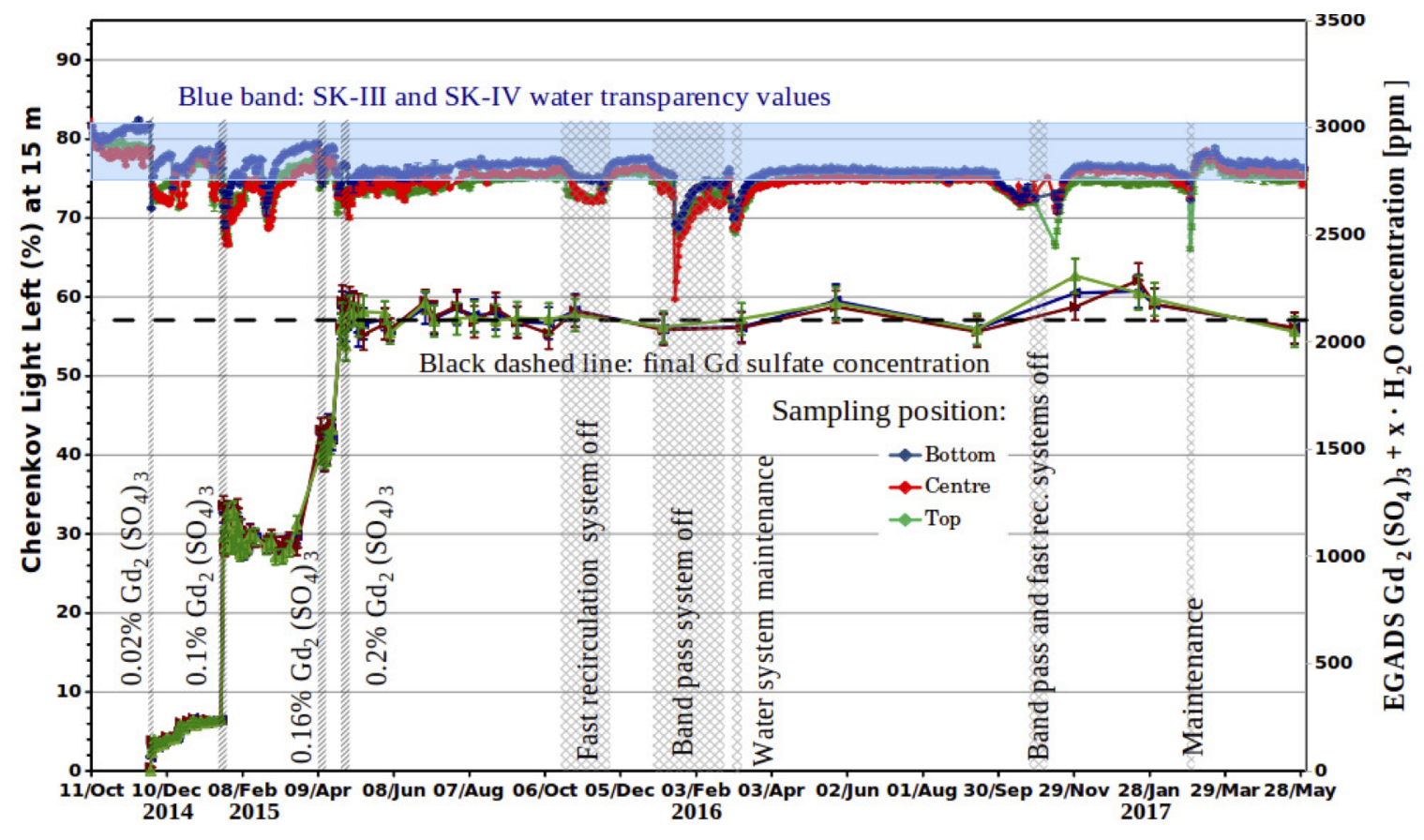

Figure 8: Water transparency in the EGADS detector. The left vertical axis and the upper red, green and blue curves indicate the Cherenkov light left after $15 \mathrm{~m}$ for three sampling positions (top, center and bottom) in the EGADS detector. The blue band indicates typical values for Super-Kamiokande. The right vertical axis and lower curves indicate the Gd sulfate concentration for the same sampling points. The black dashed line shows the final Gd sulfate concentration. Figure taken from [65].

\subsection{Super-Kamiokande and the Gadolinium upgrade}

Adding $0.2 \%$ Gadolinium sulfate to the SuperK water is a long-standing idea [66]. The extreme neutron capture cross section of Gadolinium $(\mathrm{Gd})$, together with a delayed $8 \mathrm{MeV}$ gammaray emission from the neutron capture would boost the energy threshold and background rejection capabilities to allow an observation of the SN relic background [67]. A test facility was constructed 
close to the SuperK main tank (EGADS) to test operations with Gd enriched water. In [65] the current status of the test facility is reported. Figure 8 shows that the water transparency is within nominal parameters even at the target Gd concentration. A challenge is still the radioisotope contamination introduced by the Gd. There is ongoing work with the supplier companies to mitigate this and reach the requirements for the $\mathrm{SN}$ and solar physics program.

\subsection{Prospects of Hyper-Kamiokande}

The next big step forward for MeV neutrino astronomy will be the construction of HyperKamiokande (HyperK) [68]. Technically closely related to SuperK, it would feature an approximately ten times larger fiducial interaction volume than SuperK, about $220 \mathrm{kt}$ of water. The prospects for detecting the $\mathrm{SN}$ relic background as well as the expected signals from SNe have been shown in $[69,70]$. Several tens of thousands of detected neutrinos would be expected from a SN at $10 \mathrm{kpc}$ distance. Even a SN at Andromeda would still be detectable with $\mathrm{O}(10)$ neutrinos expected. Figure 9 shows the expected time distribution of counts from a SN at $10 \mathrm{kpc}$ for different $\mathrm{SN}$ explosion models. It is clear that this data will be invaluable for improving our understanding of SN explosion physics.

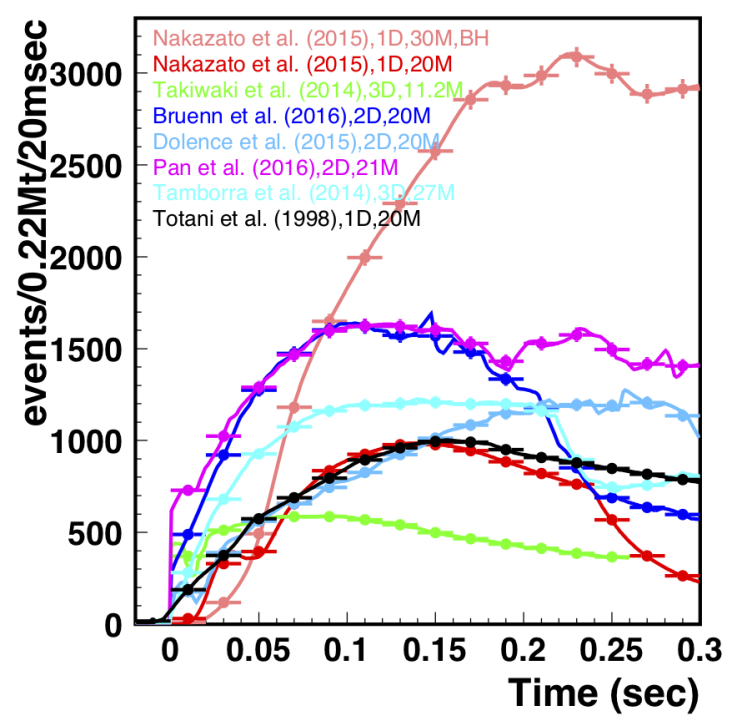

Figure 9: Event rate predicted by simulations of supernova bursts at $10 \mathrm{kpc}$ distance for the first 0.3 seconds after its onset. Figure taken from [70].

\section{New instrumentation for high-energy neutrino astrophysics}

Also in high-energy neutrino astrophysics designs for the next generation of instruments is shaping quickly, or the telescopes are already under construction. It is clear that, dependent on the nature of the cosmic neutrinos, current instruments might not be sensitive enough to resolve their origin. The strategy for the next generation is to extend the capabilities of current instruments in three directions. The under construction next generation underwater telescope KM3Net [71] 
will have a complementary view on the neutrino sky compared to IceCube with a significantly better angular resolution. The plans for an IceCube-Gen2 detector [72] surrounding IceCube at the South Pole would improve statistics and sensitivities substantially in the energy range where the cosmic neutrino flux has been observed. At high energies above tens of $\mathrm{PeV}$, radio detection of neutrino interactions in ice is progressing rapidly with several complementary approaches (ARA [73], ARIANNA [74], ANITA [53]).

\subsection{KM3NeT and Baikal-GVD}

$\mathrm{KM} 3 \mathrm{NeT}$ is an under-construction underwater neutrino telescope in the Mediterranean sea, detecting neutrinos via the Cherenkov light emission of the charged particles produced in their interactions, similar to ANTARES and IceCube. It is organized in so-called "building blocks". The high-energy array ARCA will consist of two building blocks of about $0.5 \mathrm{~km}^{3}$ instrumented volume each, i.e., have a similar volume as IceCube. ARCA is located near the coast of Sicily and so far 2 detection units have been deployed. The depth dependent muon rate measured with these detection units is shown in [75], agreeing well with expectations and confirming nominal performance. The direction reconstruction performance of KM3NeT is evaluated in [76]. Above $100 \mathrm{TeV}$ the median angular resolution for track-like events is expected to be below $0.1^{\circ}$, and below $2^{\circ}$ for shower-like events (cfr. Figure 10), a significant improvement over IceCube's angular resolution.
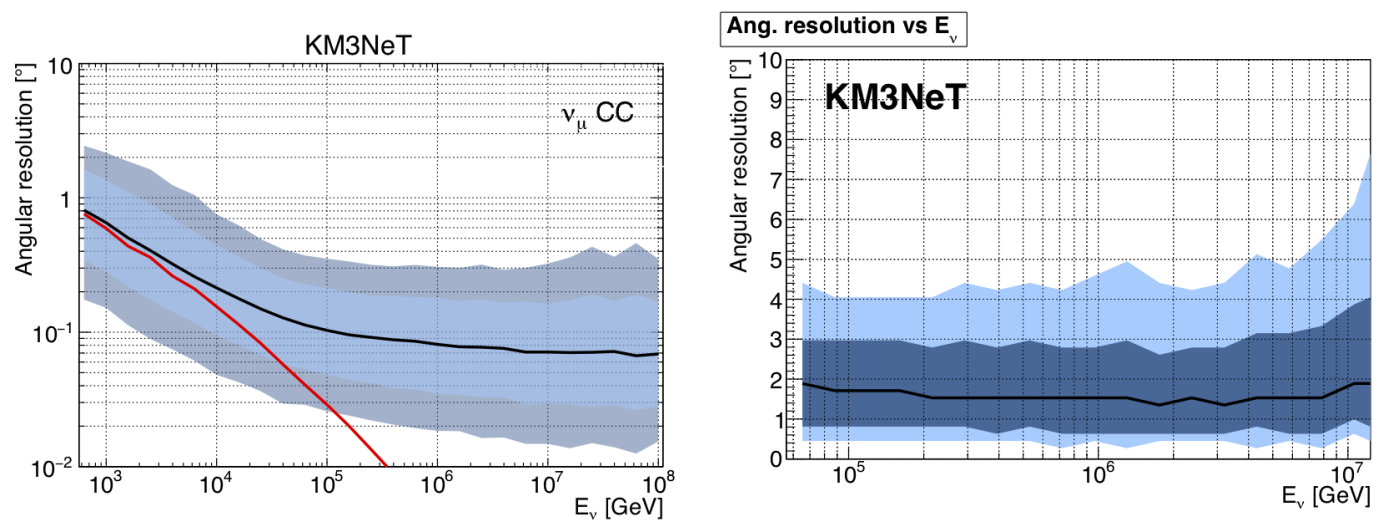

Figure 10: Left: Median angular error between reconstructed track and true neutrino direction as a function of the neutrino energy. The red line indicates the median kinematic angle between the neutrino and the secondary muon. Right: Median angular error between reconstructed shower and true neutrino direction. The colored bands represent the $68 \%$ and $90 \%$ quantiles. Figures taken from [76].

A second underwater neutrino detector on the Northern Hemisphere is currently deployed in Lake Baikal. The Gigaton Volume Detector (GVD) will eventually reach an instrumented volume of $0.4 \mathrm{~km}^{3}$, a future extension to $1.5 \mathrm{~km}^{3}$ is planned. The detector is deployed in clusters of 288 optical sensors each, and currently 2 out of 8 clusters have been deployed successfully and first observations of high-energy showers with one of the two clusters have been performed [77, 78].

\subsection{IceCube-Gen2}

IceCube-Gen2 is still in the design phase. Planned as an extension of the current IceCube 


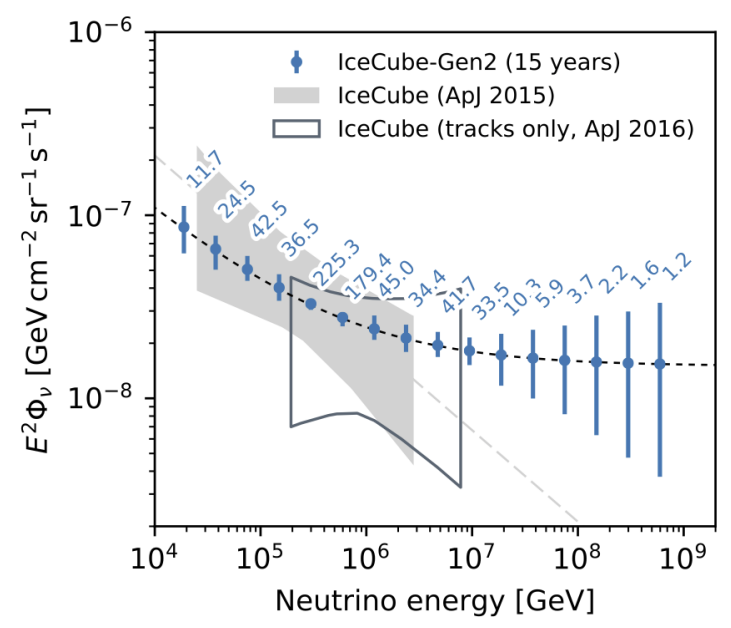

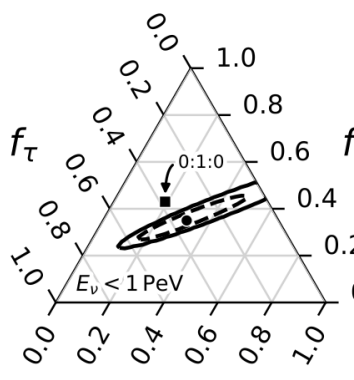

$f_{e}$

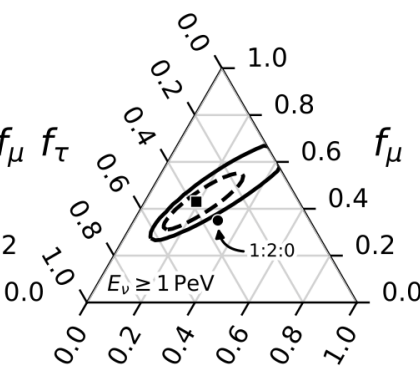

$f_{e}$

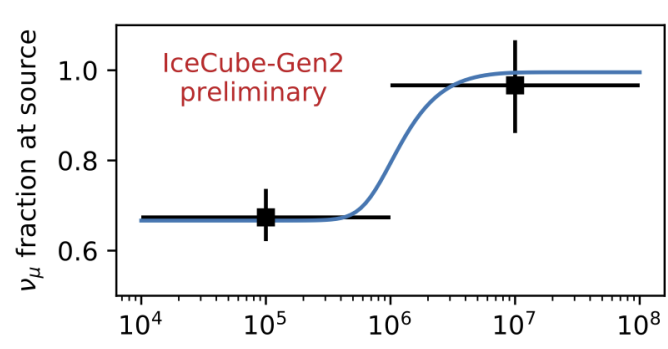

$E_{v}[\mathrm{GeV}]$

Figure 11: Upper: Expected IceCube-Gen2 diffuse neutrino flux measurement after 15 years of operation. The dotted line shows a hypothetical spectrum based on the superposition of two power-law spectra with indices $\gamma=2.5$ and $\gamma=2$, compatible with current IceCube observations (see above). The blue points and error bars show the expected precision for the measurement of such a spectrum with IceCube-Gen2. The numbers indicate the event statistics expected in each energy band. Lower: Example for the performance of IceCube-Gen2 to constrain the flavor ratio at Earth below and above $1 \mathrm{PeV}$, respectively. In the scenario presented here, the flavor composition at the source changes from $v_{e}: v_{\mu}: v_{\tau}=1: 2: 0$ to $v_{e}: v_{\mu}: v_{\tau}=0$ : $1: 0$ at a neutrino energy of $1 \mathrm{PeV}$, due to muon cooling effects at high energies. The dotted contours give the $68 \% \mathrm{CL}$ allowed region, while the solid lines correspond to $90 \% \mathrm{CL}$. The error bars in the right panel show the $68 \% \mathrm{CL}$ constraints on the muon-neutrino fraction at the source, while the line shows the injected flavor composition. Figures taken from [79].

observatory, 120 new strings with optical sensors deployed around the current IceCube and a larger horizontal spacing between the strings would increase the instrumented volume by almost an order of magnitude. In addition, a densely instrumented core for measuring neutrino properties, an extended surface array, and possibly the integration of a radio detection array (see below) is envisioned for IceCube-Gen2. The expected performance of the main array, consisting of IceCube and the 120 new strings is shown in [79]. IceCube-Gen2 will do a precision measurement of the astrophysical neutrino spectrum and be able to distinguish between different flavor composition scenarios in individual energy bands. Figure 11 shows the expected precision on the spectral measurements, as well as the flavor composition measurements below and above $1 \mathrm{PeV}$. Improved 
optical sensors are prototyped and evaluated (D-Egg [80, 81], mDOM [82]) that promise a significant improvement in sensitivity over the classic IceCube digital optical module. New calibration devices, like a camera system inside the optical module [83] to quantitatively measure the optical properties of the ice in the refrozen deployment holes will improve on systematic uncertainties.

\subsection{Radio detection of ultra-high-energy neutrinos}

The detection of radio waves from the showers produced in high-energy neutrino interactions is still a new, but very promising technology to extend the reach of neutrino observatories beyond $\mathrm{EeV}$ energies. Due to the long radio attenuation length of $\mathrm{O}(\mathrm{km})$ in glacial ice, huge target volumes in excess of $100 \mathrm{~km}^{3}$ can be instrumented cost-effectively. Currently, intensive R\&D is on-going in two test arrays in Antarctica.

The ARA detector at the South Pole has radio antennas deployed about $200 \mathrm{~m}$ deep in the ice. 3 stations are already deployed, and 3 more stations will be deployed at the end of 2017. Tests of radio pulse propagation with a radio emitter deployed on one of the IceCube strings about $3.6 \mathrm{~km}$ away from the ARA antennas confirms the ice propagation and simulation models [84]. Evidence of birefringence is seen in the radio signal. A further improvement of the ARA performance is expected from phased array triggers that will be deployed with the new ARA stations. The phased array trigger can lower the trigger threshold for an ARA-like array to $\mathrm{O}(10 \mathrm{PeV})$, substantially increasing expected event rates of astrophysical neutrinos [85].

ARIANNA is a surface radio array on the Ross Ice Shelf in Antarctica. It can detect direct radio signals from neutrino interactions, as well as signals that are reflected from the ice-sea water boundary at the bottom of the shelf. Recent measurements were reported of the radio wave propagation in the firn layer on the surface of the ice shelf that demonstrate that even far-away surface stations can observe a radio signal from a transmitter buried in the ice [86]. This would potentially allow to design surface stations for the South Pole with similar sensitivity as ARA, but at a lower cost.

\section{Conclusions}

High-energy neutrino astrophysics is a new and exciting field that gives us a first glimpse into a yet to be explored uinverse. The spectrum and flavor composition is measured with increasing precision as IceCube collects more data. Yet, no sources have been identified by either IceCube or ANTARES. However, non-observations constrain the contributions of several source populations to the total astrophysical neutrino flux. While the neutrino flux is observed from about $10 \mathrm{TeV}$ to several PeV, no clear evidence for a signal from cosmogenic neutrinos at $\mathrm{EeV}$ energies is seen by either IceCube, the PAO or the radio detectors ANITA, ARA and ARIANNA. Modeling efforts continue to connect physics processes at the sources to observations, and describe the background of atmospheric neutrinos with better precision.

$\mathrm{MeV}$ astronomy with neutrinos is established since many years, focusing on the sun and $\mathrm{SNe}$ as sources. High-statistics observations of the neutrino flux from the sun allow a detailed analysis of the time structure of the emission. An effort to add Gadolinium sulfate to the SuperK detector promises to facilitate the detection of the supernova relic background. And, at any time, a Galactic supernova explosion could give us unprecedented statistics on supernova neutrinos from a network 
of detectors around the world. HyperK with an order of magnitude larger fiducial volume will deliver another large statistics increase rendering even $\mathrm{SNe}$ at the Andromeda galaxy detectable.

Finally, strong efforts go into the development of the next generation of instruments. A new generation of underwater neutrino telescopes (KM3Net, Baikal-GVD) is already under construction and will allow complementary observations of the neutrino sky with higher angular resolution. The planned IceCube-Gen2 detector will provide a high-precision measurement of the cosmic neutrino spectrum and flavor composition, and a several times better sensitivity to neutrino sources. Radio detection of neutrino interactions is still a new, but fast evolving technology that will in the near future allow to cost-effectively instrument hundreds of cubic kilometers of ice target for the observation of neutrinos at EeV energies.

\section{References}

[1] IceCube Collaboration, M. G. Aartsen et al., Science 342 (2013) 1242856.

[2] J. N. Bahcall, Phys. Rev. Lett. 12 (1964) 300-302.

[3] R. Davis, Phys. Rev. Lett. 12 (1964) 303-305.

[4] Kamiokande-II Collaboration, K. Hirata et al., Phys. Rev. Lett. 58 (1987) 1490-1493.

[5] IceCube Collaboration, M. G. Aartsen et al., Astrophys. J. 809 (2015) 98.

[6] Fermi-LAT Collaboration, M. Ackermann et al., Science 339 (2013) 807.

[7] IceCube Collaboration, M. G. Aartsen et al., JINST 12 (2017) P03012.

[8] IceCube Collaboration, PoS ( ICRC2015) 1066 (2016).

[9] IceCube Collaboration, PoS ( ICRC2017) 981 (2017).

[10] IceCube Collaboration, PoS ( ICRC2017) 968 (2017).

[11] IceCube Collaboration, PoS ( ICRC2017) 1005 (2017).

[12] IceCube Collaboration, PoS ( ICRC2 017 ) 976 (2017).

[13] IceCube Collaboration, PoS ( ICRC2017) 974 (2017).

[14] IceCube Collaboration, PoS ( ICRC2017) 973 (2017).

[15] IceCube Collaboration, PoS (ICRC2017) 1002 (2017).

[16] IceCube Collaboration, PoS ( ICRC2017) 1008 (2017).

[17] S. W. Li, M. Bustamante, and J. F. Beacom, arXiv:1606.06290.

[18] ANTARES Collaboration, M. Ageron et al., Nucl. Instrum. Meth. A656 (2011) 11-38.

[19] Pierre Auger Collaboration, A. Aab et al., Nucl. Instrum. Meth. A798 (2015) 172-213.

[20] Pierre Auger Collaboration, PoS ( ICRC2017) 972 (2017).

[21] ANTARES Collaboration, POS ( ICRC2017) 986 (2017).

[22] IceCube Collaboration, PoS ( ICRC2017) 963 (2017).

[23] IceCube Collaboration, M. G. Aartsen et al., Astrophys. J. 835 (2017) 151.

[24] IceCube Collaboration, M. G. Aartsen et al., Astrophys. J. 824 (2016) L28. 
[25] Virgo, LIGO Scientific Collaboration, B. P. Abbott et al., Phys. Rev. Lett. 116 (2016) 061102.

[26] ANTARES Collaboration, POS ( ICRC2017) 988 (2017).

[27] ANTARES Collaboration, POS ( ICRC2017) 970 (2017).

[28] ANTARES Collaboration, PoS ( ICRC2017) 946 (2017).

[29] IceCube Collaboration, PoS ( ICRC2 017 ) 957 (2017).

[30] IceCube, FACT, MAGIC Collaboration, P OS ( ICRC2017) 969 (2017).

[31] ANTARES Collaboration, PoS ( ICRC2017) 989 (2017).

[32] IceCube Collaboration, PoS ( ICRC2017) 980 (2017).

[33] ANTARES Collaboration, PoS ( ICRC2017) 947 (2017).

[34] IceCube Collaboration, PoS ( ICRC2017) 1015 (2017).

[35] ANTARES Collaboration, POS ( ICRC2017) 971 (2017).

[36] IceCube Collaboration, PoS ( ICRC2017) 1007 (2017).

[37] ANTARES Collaboration, POS ( ICRC2017) 987 (2017).

[38] Fermi-LAT Collaboration, M. Ackermann et al., Astrophys. J. 810 (2015) 14.

[39] Fermi-LAT Collaboration, M. Ackermann et al., Astrophys. J. Suppl. 222 (2016) 5.

[40] Y. L. Chang, B. Arsioli, P. Giommi, and P. Padovani, Astron. Astrophys. 598 (2017) A17.

[41] IceCube Collaboration, POS ( ICRC2017) 994 (2017).

[42] IceCube Collaboration, PoS ( ICRC2017) 1014 (2017).

[43] ANTARES Collaboration, POS ( ICRC2017) 949 (2017).

[44] IceCube, Pierre Auger, Telescope Array Collaboration, P OS ( ICRC2017) 961 (2017).

[45] M. Fukushima, Prog. Theor. Phys. Suppl. 151 (2003) 206-210.

[46] IceCube, Pierre Auger, Telescope Array Collaboration, M. G. Aartsen et al., JCAP 1601 (2016) 037.

[47] ANTARES Collaboration, PoS ( ICRC2017) 990 (2017).

[48] IceCube Collaboration, PoS ( ICRC2017) 995 (2017).

[49] IceCube Collaboration, P OS ( ICRC2017) 1011 (2017).

[50] ANTARES Collaboration, POS ( ICRC2017) 942 (2017).

[51] D. Gaggero, D. Grasso, A. Marinelli, A. Urbano, and M. Valli, Astrophys. J. 815 (2015) L25.

[52] IceCube Collaboration, POS ( ICRC2017) 975 (2017).

[53] ANITA Collaboration, P. W. Gorham et al., Astropart. Phys. 32 (2009) 10-41.

[54] ANITA Collaboration, PoS ( ICRC2017) 935 (2017).

[55] D. Boncioli, D. Biehl, A. Fedynitch and W. Winter, PoS ( ICRC2017) 1064 (2017).

[56] W. Bednarek, POS (ICRC2017) 1069 (2017).

[57] K. Murase, D. Guetta, and M. Ahlers, Phys. Rev. Lett. 116 (2016) 071101.

[58] M. Honda, T. Kajita, K. Kasahara, S. Midorikawa and J. Nishimura, PoS ( ICRC2 017 ) 1022 (2017). 
[59] AMS Collaboration, M. Aguilar et al., Phys. Rev. Lett. 114 (2015) 171103.

[60] AMS Collaboration, M. Aguilar et al., Phys. Rev. Lett. 115 (2015) 211101.

[61] A. Fedynitch, H. Dembinski, R. Engel, T.K. Gaisser, F. Riehn and T. Stanev, POS ( ICRC2017) 1019 (2017).

[62] H. Dembinski, R. Engel, A. Fedynitch, T.K. Gaisser, F. Riehn and T. Stanev, PoS ( ICRC2017) 533 (2017).

[63] Super-Kamiokande Collaboration, Y. Fukuda et al., Nucl. Instrum. Meth. A501 (2003) 418-462.

[64] T. Yano, PoS (ICRC2017) 1066 (2017).

[65] Super-Kamiokande Collaboration, POS ( ICRC2017) 1043 (2017).

[66] J. F. Beacom and M. R. Vagins, Phys. Rev. Lett. 93 (2004) 171101.

[67] S. Horiuchi, J. F. Beacom, and E. Dwek, Phys. Rev. D79 (2009) 083013.

[68] K. Abe et al., arXiv:1109.3262.

[69] Hyper-Kamiokande Collaboration, PoS ( ICRC2017) 948 (2017).

[70] T. Yano, PoS ( ICRC2017) 954 (2017).

[71] KM3Net Collaboration, S. Adrian-Martinez et al., J. Phys. G43 (2016) 084001.

[72] IceCube Collaboration, M. G. Aartsen et al., arXiv:1412.5106.

[73] P. Allison et al., Astropart. Phys. 35 (2012) 457-477.

[74] S. W. Barwick et al., Astropart. Phys. 90 (2017) 50-68.

[75] KM3NeT Collaboration, PoS ( ICRC2017) 1018 (2017).

[76] KM3NeT Collaboration, PoS ( ICRC2017) 950 (2017).

[77] Baikal-GVD Collaboration, PoS ( ICRC2017) 962 (2017).

[78] Baikal-GVD Collaboration, PoS1034 ( ICRC2017) 1034 (2017).

[79] IceCube-Gen2 Collaboration, PoS ( ICRC2017) 991 (2017).

[80] IceCube-Gen2 Collaboration, P OS ( ICRC2017) 1051 (2017).

[81] IceCube-Gen2 Collaboration, P OS ( ICRC2017) 1038 (2017).

[82] IceCube-Gen2 Collaboration, P OS ( ICRC2017) 1047 (2017).

[83] IceCube-Gen2 Collaboration, POS ( ICRC2017) 1040 (2017).

[84] ARA Collaboration, PoS ( ICRC2017) 1030 (2017).

[85] ARA Collaboration, PoS ( ICRC2017) 1013 (2017).

[86] ARIANNA Collaboration, PoS ( ICRC2017) 1042 (2017). 